\title{
Passing the DVLA field regulations following bilateral macular photocoagulation in diabetics
}

\begin{abstract}
Purpose At present the Driver and Vehicle and Licensing Agency (DVLA) of the UK requires all diabetic patients with laser treatment in both eyes to undergo a binocular driving field assessment. Increasingly we find that many such patients have undergone only macular laser treatment without panretinal photocoagulation. We aimed to ascertain the frequency and causes of failure of the binocular driving field test after bilateral macular laser treatment performed according to a set protocol.
\end{abstract}

Methods A retrospective case notes study was combined with a prospective recall of patients for visual field assessment. All diabetic patients under the care of one consultant who had had bilateral macular laser treatment (without panretinal photocoagulation) over a 4 year period were identified and the case notes studied. Patients who could not achieve a corrected binocular acuity of $6 / 9$ were excluded, as were patients with glaucoma. Patients who had not had the DVLA test were recalled to perform the test, whether or not they had an intention to drive. Failure was defined utilising strict criteria on the Esterman Programme of the Humphrey Analyser.

Results A total of 138 patients were identified. Fifty-four were not eligible ( 21 because of poor acuity, 29 had died or were too ill to participate and 4 for other reasons). Of the 84 who were eligible, 64 had visual fields performed and 20 declined to attend. Sixty-three of 64 tested passed the Esterman test, the one failure having a previously unknown and relatively mild homonymous field defect.

Conclusion Patients undergoing bilateral macular laser can be counselled that, provided their acuity remains adequate, they have a very low chance of failing the DVLA field regulations.

Key words Binocular visual fields, Diabetic maculopathy, Driving, Laser
Maculopathy is relatively common in diabetics, particularly those with type II disease. ${ }^{1}$ Macular laser treatment has been shown to preserve the acuity of many of these patients. ${ }^{2}$ At present any patient who has had laser treatment in both eyes is required by the Driver and Vehicle Licensing Agency (DVLA) of the UK to undergo a binocular field test. ${ }^{3}$ Whilst it is well known that patients who have had bilateral panretinal photocoagulation (PRP) may fail such a test, ${ }^{4-6}$ and bilateral paracentral scotomata following macular laser treatment can cause a failure, ${ }^{6}$ no case series of patients who have undergone macular treatment without PRP has, to our knowledge, been studied.

The purpose of this study was to ascertain the frequency and reasons for any failure of binocular driving fields after bilateral macular laser treatment performed according to a set protocol by a team working in a diabetic retinal clinic.

\section{Patients and methods}

The patients who had undergone bilateral macular laser treatment, but no PRP, under a single consultant's care between April 1994 and April 1998 were identified from the laser register and their case notes subjected to study.

In cases where the binocular visual acuity was not recorded in the case notes, patients with a monocular best corrected visual acuity of $6 / 12$ or better in each eye of $6 / 9$ or better in one eye at the last clinic visit were included. These patients were included because they were likely to pass the visual acuity criteria for driving set by DVLA. (In the clinic situation this translates to a binocular Snellen visual acuity of $6 / 10$ or better in the best possible lighting.)

Macular laser was defined here as argon retinal laser photocoagulation applied to areas within a disc diameter from the centre of the fovea and possibly outside this area but not anywhere peripheral to the temporal arcades. This may be photocoagulation of microaneurysms or grid laser treatment to areas of retinal oedema or both. Grid laser involves
L. Tong

S.A. Vernon Department of Ophthalmology Queen's Medical Centre University Hospital Nottingham, UK

Mr S.A. Vernon Ophthalmology Directorate Queen's Medical Centre University Hospital Nottingham NG7 2UH, UK

Proprietary interest: None

Grant support: None 


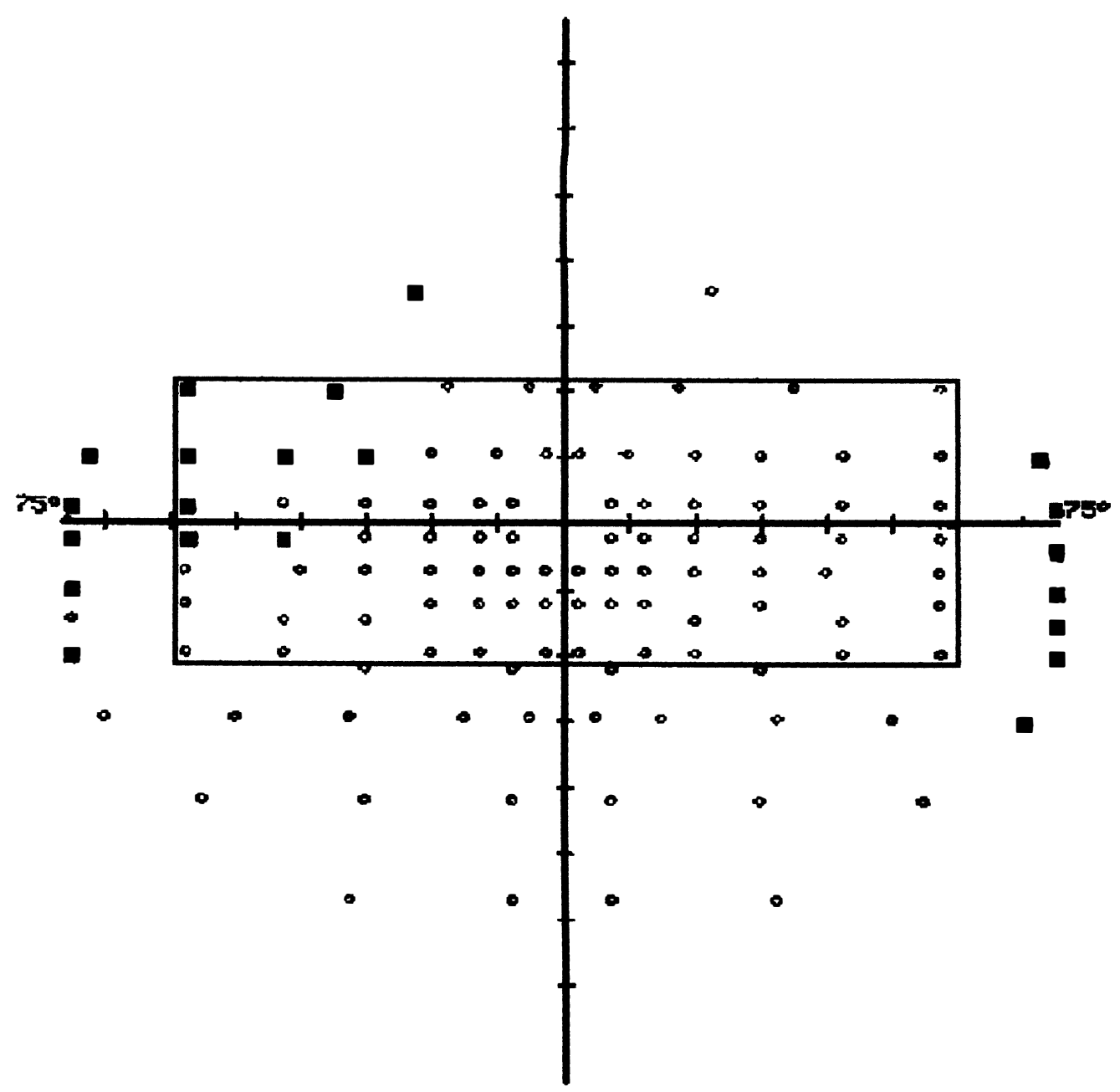

Fig. 1. The Esterman binocular visual field. Open circles, points seen (99/120); filled squares, points missed (21/120).

spacing burns about one burn apart over oedematous retina and burns over microaneurysms may be repeated to achieve blanching whenever necessary.

Since all the patients in this study were treated in the same consultant team a uniform strategy was adopted for treatment. In particular we have a policy not to perform confluent ablative treatment. All the patients were treated using an argon laser with a fundus contact lens which does not significantly magnify the spot size of the laser beam. The indications for treatment were defined by the EDTRS criteria. ${ }^{2}$ Retreatment was allowable when considered necessary. The total number of burns applied, the duration of exposure of each burn, spot size and power setting were recorded on a database. Any patients not passing the visual acuity criteria set of DVLA (as defined above) were noted.

The results of the Esterman driving visual field assessment done on those patients who had already performed it were noted. The remaining patients who for any reason had not had the test were contacted and requested to perform it following suitable explanation. The records of patients who declined to attend were examined and their laser details compared with all those who had performed the test.
All binocular driving visual field tests were performed with the Humphrey field analyser using a 121 point Esterman strategy. Points considered relevant extend over a horizontal extent of $120^{\circ}$ and $40^{\circ}$ vertically centred on fixation. Standard settings for the DVLA test were employed. No optical correction was used during the test, as is usual practice in our unit. The test was performed once and patients recording a marginal failure (defined as one missed point within the field of interest defined above) underwent a repeat test.

We defined a pass as a positive response to all Humphrey targets within the $120^{\circ} \times 40^{\circ}$ area marked on Fig. 1. For the purposes of this study any missed point(s) within this zone constituted a failure (allowing a repeat test for one missed point as described above).

\section{Results}

One hundred and thirty-eight patients had had bilateral laser therapy for diabetic maculopathy (without PRP) during the 4 year study period. Fifty-four patients were not eligible ( 24 had died during the study period, 21 patients were excluded because of poor acuity, none of which had worse than counting fingers in one eye, 1 had glaucoma coexisting with diabetic maculopathy, 5 were 


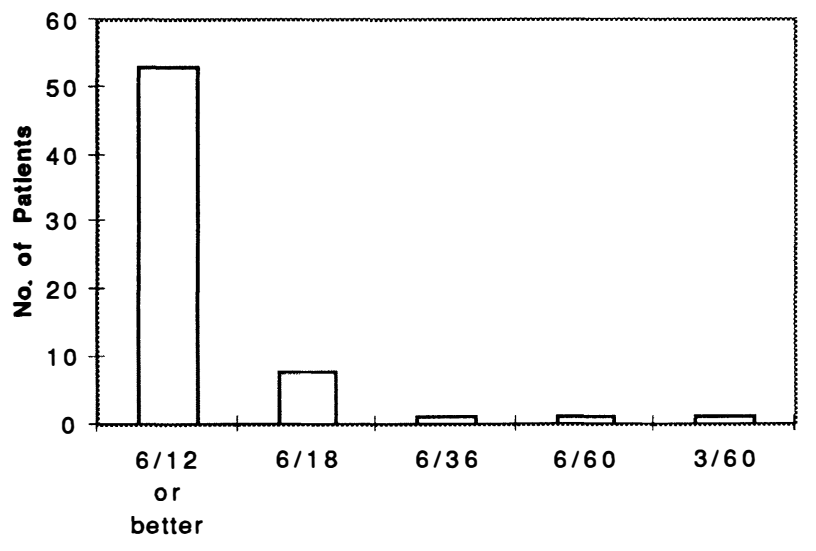

Visual Aculty

Fig. 2. Corrected visual acuity of the worse eye in patients who performed the visual field test.

too sick to participate, 2 had moved out of the region, and 1 set of case notes was missing). Eighty-four patients were therefore eligible. Nineteen patients had had Esterman fields previously (none has had further laser since the field was performed) and 65 patients were recalled. Of the 65 recalled 20 refused to participate for varying reasons. At the time of the last clinic review, none of the eligible patients had clinical maculopathy which required more photocoagulation.

In the 64 patients who had visual fields done, the mean age was 63 years (range 34-81 years, standard deviation 11); Fig. 2 shows the distribution of the best corrected monocular visual acuity in the eye with the worse visual acuity. In these patients the mean number of laser sessions performed prior to the test in each eye was 2.06. Argon green wavelength was used in all cases. The mean total number of burns was (right eye)

$173 /$ (left eye) 176 , and the mean mean power setting was (RE) $0.24 /$ (LE) $0.25 \mathrm{~W}$. The spot size used averaged (RE)106/(LE)108 $\mu \mathrm{m}$ diameter. The duration of exposure per photocoagulation burn was $0.1 \mathrm{~s}$ in all cases.

All the patients who declined to attend the visual field test claimed to be non-drivers on questioning. Table 1 summarises the treatment parameters of those patients who did and did not perform the field test. There was no significant difference in any of the parameters between the two groups on unpaired two-tailed $t$-test at a significance level of $p=0.05$.

Sixty-three of the 64 patients who took the Esterman test passed, with 4 of those who passed requiring a second test due to a missing single point on the first test. The one patient who failed was later identified to have a relatively mild homonymous hemianopic defect. He had passed on the points in the central $20^{\circ}$ of fixation where misses would be expected to occur when secondary to laser therapy (Fig. 1).

\section{Discussion}

The importance of a good binocular visual field in the context of driving has been previously documented. ${ }^{7}$ Constriction of this field can cause reduced ability to identify road signs and avoid obstacles, and can increase the time needed to complete a driving course. ${ }^{7}$ To date there has been no published literature on the effects of isolated paracentral scotomata on driving, but these may be used by the DVLA as a reason for failure. ${ }^{8}$

There is well-documented evidence of loss of uniocular visual field after bilateral PRP in diabetics. ${ }^{8-12}$ High energy used during coagulation may cause more visual field losses. ${ }^{12}$

A number of studies have been performed with respect to binocular fields (according to DVLA criteria) following PRP, with one study recording a $50 \%$ failure rate of the DVLA regulations. ${ }^{4}$ Interestingly 2 patients who failed had only had unilateral PRP, and the failure was attributed to field losses unrelated to laser treatment. Other studies have shown that bilateral PRP can lead to a much lower frequency of DVLA field failure, particularly when smaller spot sizes are used. ${ }^{6,13}$ More recently, it has been suggested that a $12 \%$ failure rate may be expected after bilateral PRP, the risk being greater in type II diabetics. ${ }^{10}$ In addition, there may be substantial differences in the assessment of driving fields between consultants and the Visual Standards Sub-committee (VSSC), with more cases passing when the fields were assessed by the VSSC. ${ }^{9}$

Previous studies have documented a decrease in retinal sensitivity after laser treatment of the macula when uni-ocular fields were assessed. Central $30^{\circ}$ visual field sensitivity has been noted to decrease after diode, YAG and krypton laser treatment in diabetics with maculopathy. ${ }^{14}$ Perifoveal laser treatment of the macula for subretinal membranes can lead to relative scotomata which increase in depth over time ${ }^{15}$ However, this type of treatment is usually designed to be confluent in nature and this effect may not be clinically relevant following commonly used diabetic maculopathy treatment strategies.

Diabetic retinopathy itself may lead to uni-ocular visual field losses. ${ }^{16}$ Using a moving target at a distance of $1 \mathrm{~m}$, scotomata subtending half a degree angle can be identified. Relative scotomata were observed in diabetics with little or no retinopathy and absolute scotomata in

Table 1. Laser parameters in patients who performed the Esterman field test and those who did not (standard deviation in parentheses)

\begin{tabular}{|c|c|c|c|c|}
\hline & \multicolumn{2}{|c|}{ Patients who came for field test } & \multicolumn{2}{|c|}{ Patients who did not come for field test } \\
\hline & Right eye & Left eye & Right eye & Left eye \\
\hline No. of treatment sessions & $2.06(1.14)$ & $2.06(0.99)$ & $1.45(0.31)$ & $1.37(0.29)$ \\
\hline Total no. of spots over sessions & $173(148)$ & $176(135)$ & $181(180)$ & $214(181)$ \\
\hline Mean spot diameter (mm) & $106(20)$ & $108(23)$ & $113(25.5)$ & $115(29.1)$ \\
\hline Mean duration per spot (s) & 0.10 & 0.10 & 0.10 & 0.10 \\
\hline
\end{tabular}


those with severe retinopathy. It was not certain what the exact target brightness was over the background, and the target sizes used in Roth's study were smaller than those used in the Esterman binocular field. All 31 eyes of 21 patients studied were reported to have scotomata. Modern computerised techniques have revealed uniocular field defects in untreated diabetic retinopathy. ${ }^{17,18}$ The grade of retinopathy may affect the incidence of visual field loss. ${ }^{18}$ Diabetic children without retinopathy may also show field losses. ${ }^{19}$

There is, however, no evidence in the literature that retinopathy per se can lead to significant losses (in this context) in the binocular visual field, as defects in one eye tend to be compensated by a normal field in the other.

Other possible reasons for visual field losses in patients with primary diabetes mellitus include older age, cataracts, ${ }^{20}$ age-related macular degeneration, ${ }^{21,22}$ glaucoma, ${ }^{23}$ optic neuropathy and acute hypoglycaemia. ${ }^{24}$ We only excluded known cases of glaucoma in our study, thereby including the vast majority of those currently taking the DVLA test following bilateral macular laser therapy.

As many patients in the UK undergo bilateral macular laser without PRP, the performance of driving visual fields on all these patients represents a significant use of resources. Time and manpower are required to order, perform and process the results of the tests. Although not formally measured, much anxiety is also engendered in those instructed to undergo the test procedure, as failure may have significant effects on lifestyle.

The results of this study suggest that binocular visual fields may not need to be a routine part of the assessment of patients who drive and who have had bilateral laser treatment for diabetic maculopathy. Further studies will be necessary to determine whether the test should be performed in patients who have theoretical risk factors for failing the test, i.e. those blind in one eye and those who have had very extensive treatment where the number, size of burn and confluency differ significantly from those found in our study. However, at present, patients similar to those studied may be counselled with some confidence that their chances of failing the DVLA field test are very low.

\section{References}

1. Bailey CC, Sparrow JM, Grey RHB, Cheng H. The National Diabetic Retinopathy Laser Treatment Audit. I.

Maculopathy. Eye 1998;12:69-76.

2. Early Treatment Diabetic Retinopathy Study Report Number 1. Arch Ophthalmol 1985;103:1796-806.

3. Communication to drivers from the Driver and Vehicle and Licensing Agency (DVLA).
4. Buckley SA, Jenkins L, Benjamin L. Fields, DVLC and panretinal photocoagulation. Eye 1992;6:623-5.

5. Frier BM. Driving and diabetes. BMJ 1992;305:1238.

6. Hulbert MFG, Vernon SA. Passing the DVLA field regulations following bilateral pan-retinal photocoagulation in diabetics. Eye 1992;6:456-60.

7. Wood JM, Troutbeck R. Effect of restriction of the binocular visual field on driving performance. Ophthalmic Physiol Opt 1992;12:291-8.

8. Visual standards for driving. London: Royal College of Ophthalmologists, 1999.

9. Pearson AR, Keightley SJ, Casswell AG. How good are we at assessing driving visual fields in diabetes? Eye 1998;12:938-42.

10. Pearson AR, Tanner V, Keightley SJ, Caswell AG. What effect does laser photocoagulation have on driving visual fields in diabetes? Eye 1998;12:64-8.

11. Seiberth V, Alexandridis E, Feng W. Function of the diabetic retina after panretinal argon laser coagulation. Graefes Arch Clin Exp Ophthalmol 1987;225:385-90.

12. Seiberth V, Alexandridis E. Function of the diabetic retina after pan-retinal argon laser photocoagulation: influence of the intensity of the coagulation spots. Ophthalmologia 1991;202:10-7.

13. Mackie SW, Webb LA, Hutchinson BM, Hammer HM, Barrie T, Walsh G. How much blame can be placed on laser photocoagulation for failure to attain driving standards? Eye 1995;9:517-25.

14. Okuyama M, Okisaka S, Ito M. Comparative study on frequency-doubled Nd:YAG laser, krypton laser and diode laser photocoagulation for diabetic maculopathy. Nippon Gauka Gakkai Zasshi 1995;99:87-92.

15. Schneider U, Kuck H, Kreissig I. Fixation and central visual field after perifoveal krypton laser treatment of subfoveal neovascularisations. Eur J Ophthalmol 1993;3:193-200.

16. Roth JA. Central visual fields in diabetes. $\mathrm{Br} \mathrm{J}$ Ophthalmol 1969;53:16-25.

17. Trick GL, Trick LR, Kilo C. Visual field defects in patients with insulin-dependent and non-insulin dependent diabetes. Ophthalmology 1990;97:475-82.

18. Henricsson M, Heijl A. Visual fields at different stages of diabetic retinopathy. Acta Ophthalmol (Copenh) 1994;72:560-9.

19. Mastropasqua L, Verrotti A, Lobefalo L, Chiarelli F, Verdesca G, Morgese G. Visual field defects in diabetic children without retinopathy: relation between visual function and microalbuminuria. Acta Ophthalmol Scand 1995;73:125-8.

20. Lutze M, Bresnick GH. Lens-corrected visual field sensitivity and diabetes. Invest Ophthalmol Vis Sci 1994;35:649-55.

21. Midena E, Segato T, Blarzino MC, Degli Angeli C. Macular drusen and the sensitivity of the central visual field. Doc Ophthalmol 1994;88:179-85.

22. Tolentino MJ, Miller S, Gaudio AR, Sandberg MA. Visual field deficits in early age-related macular degeneration. Vision Res 1994;34:409-13.

23. Klein BE, Klein R, Jensen SC. Open-angle glaucoma and older-onset diabetes. The Beaver Dam Eye Study. Ophthalmology 1994;101:1173-7.

24. Mosier MA, Deshmukh SS. Retinal sensitivity in acute hypoglycemia. Ophthalmologica 1990;201:99-103. 